\title{
Optical conductivity of the half-filled Hubbard chain
}

\author{
E. Jeckelmann and F. Gebhard \\ Fachbereich Physik, Philipps-Universität Marburg, D-35032 Marburg, Germany \\ F.H.L. Essler \\ Department of Physics, Warwick University, Coventry, CV4 7AL, UK
}

\begin{abstract}
We combine well-controlled analytical and numerical methods to determine the optical conductivity of the one-dimensional Mott-Hubbard insulator at zero temperature. A dynamical density-matrix renormalization group method provides the entire absorption spectrum for all but very small coupling strengths. In this limit we calculate the conductivity analytically using exact field-theoretical methods. Above the Lieb-Wu gap the conductivity exhibits a characteristic square-root increase. For small to moderate interactions, a sharp maximum occurs just above the gap. For larger interactions, another weak feature becomes visible around the middle of the absorption band.
\end{abstract}

PACS numbers: 71.10.Fd, 72.80.Sk

In quasi one-dimensional materials like, e.g., organic semiconductors [1], the electron-electron interaction accounts for the formation of excitons and, to a substantial degree, for the optical gap itself [2]. Therefore, a microscopic theory of the optical absorption in these materials requires a detailed understanding of one-dimensional Mott insulators [3].

The paradigm for a one-dimensional Mott insulator is the half-filled Hubbard model [4]

$$
\hat{H}=-t \sum_{l ; \sigma}\left(\hat{c}_{l, \sigma}^{+} \hat{c}_{l+1, \sigma}+\text { h.c. }\right)+U \sum_{l} \hat{n}_{l, \uparrow} \hat{n}_{l, \downarrow} .
$$

It describes $\pi$ electrons with spin $\sigma=\uparrow, \downarrow$ which move from one site $l$ of the carbon backbone to its neighboring sites. The lattice spacing is set to unity. The number of electrons $N$ equals the number of lattice sites $L$. Using periodic boundary conditions, the kinetic energy is diagonal in momentum space and gives rise to a cosine band, $\epsilon(k)=-2 t \cos (k)$ of width $W=4 t$. The electrons' mutual Coulomb repulsion is mimicked by the purely local Hubbard interaction $U$.

The model (1) is Bethe-Ansatz solvable [5]. The optical gap is given by 6 .

$$
\Delta(U)=\frac{16 t^{2}}{U} \int_{1}^{\infty} \frac{d y \sqrt{y^{2}-1}}{\sinh (2 \pi t y / U)} .
$$

For small and large $U / t$ this gives $\Delta(U \lesssim 2 t)=$ $(8 t / \pi) \sqrt{U / t} \exp (-2 \pi t / U)$ and $\Delta(U \gtrsim 4 t)=U-W+$ $8 \ln (2) t^{2} / U$. The optical absorption is proportional to the real part of the optical conductivity which is related to the imaginary part of the current-current correlation function by $\sigma_{1}(\omega>0)=\operatorname{Im}\left\{\chi_{j j}(\omega>0)\right\} / \omega$, and

$$
\begin{aligned}
\chi_{j j}(\omega>0) & =-\frac{1}{L}\left\langle 0\left|\hat{\jmath} \frac{1}{E_{0}-\hat{H}+\hbar \omega+i \eta} \hat{\jmath}\right| 0\right\rangle \\
& =-\frac{1}{L} \sum_{n} \frac{|\langle 0|\hat{\jmath}| n\rangle|^{2}}{\hbar \omega-\left(E_{n}-E_{0}\right)+i \eta} .
\end{aligned}
$$

Here, $|0\rangle$ is the ground state, $|n\rangle$ are excited states, and $E_{0}, E_{n}$ are their respective energies. Although $\eta=0^{+}$is infinitesimal, we may introduce a finite value to broaden our resonances at $\hbar \omega=E_{n}-E_{0}$. In momentum space, $\hat{\jmath}=-(2 e t / \hbar) \sum_{k ; \sigma} \sin (k) \hat{c}_{k, \sigma}^{+} \hat{c}_{k, \sigma}$, is the current operator. We set $\hbar=1$ throughout, and for our numerical results we use $e=t \equiv 1$ in our figures.

Eq. (3) shows why it is so difficult to calculate optical properties of Mott-Hubbard insulators. The spectrum $E_{n}$ is known exactly but very little is known about the oscillator strengths $T_{0, n}^{2}=|\langle 0|\hat{\jmath}| n\rangle|^{2}$, although it can be shown explicitly that they vanish unless $E_{n}-E_{0} \geq$ $\Delta$ [7]. Numerical calculations of $\sigma_{1}(\omega)$ have been carried out using exact diagonalizations [8] and quantum Monte Carlo simulations [9]. Unfortunately, these approaches are seriously limited in accuracy or accessible system sizes. Since the system is a Mott insulator and not a Luttinger liquid metal, standard bosonization techniques cannot be applied to the half-filled Hubbard model [10]. Therefore, the calculation of the optical conductivity of this model is an important yet unsolved problem in theoretical solid-state physics.

In this work we employ the dynamical density-matrix renormalization group (DDMRG) method 11, 12 to determine the optical conductivity of the Hubbard insulator over the entire absorption spectrum. This numerical technique allows us to obtain $\sigma_{1}(\omega)$ for all interaction strengths as long as the gap is not exponentially small, $U \gtrsim 3 t$. For large interaction strengths, $U \gg t$, we confirm results obtained in the framework of a $1 / U$ expansion 13. In the weak-coupling regime $(\Delta \ll t)$, we calculate $\sigma_{1}(\omega)$ analytically using exact field-theoretical methods. The analytical results agree well with the DDMRG data for small $U / t$.

We start our analysis with the large- $U$ limit $(U / t \rightarrow$ $\infty)$, where a rather simple band picture emerges [13]. If we ignore corrections of the order $t / U$ electron transfers are limited to those processes which conserve the number 
of double occupancies. Due to spin-charge separation, the oscillator strength $T_{0, n}^{2}$ can be written as the convolution of the charge and spin contributions. The charge contribution follows from a simple band picture: we excite one hole in the lower Hubbard band, $\epsilon_{\mathrm{LHB}}(k)=\epsilon(k)$, and one double occupancy in the upper Hubbard band, $\epsilon_{\mathrm{UHB}}(k)=U-\epsilon(k)$ (antiparallel bands). The total momentum of the two charge excitations is $q$, and their energy is $\omega$. The spin sector enters the current-current correlation function via the momentum-dependent groundstate form-factor $g_{q}$.

For the large- $U$ Hubbard model itself $(U / t \rightarrow \infty)$, the analysis of $g_{q}$ is rather involved. However, explicit analytical results are available for the closely related problems of a dimerized (DIM) or a Néel-ordered (AF) spin ground state. For these states, $g_{q}$ vanishes unless $q=0$ or $q=\pi$, $g_{0}^{\mathrm{AF}}=2, g_{\pi}^{\mathrm{AF}}=0$, and $g_{0}^{\mathrm{DIM}}=9 / 4, g_{\pi}^{\mathrm{DIM}}=1 / 4$ 13. Now, optical transitions occur between two antiparallel bands $\left(q=0 ; \epsilon_{\mathrm{LHB}}(k), \epsilon_{\mathrm{UHB}}(k)\right)$ and between two parallel bands $\left(q=\pi ; \epsilon_{\mathrm{LHB}}(k), \epsilon_{\mathrm{UHB}}(k+\pi)\right)$. Then, the optical conductivity becomes

$$
\begin{aligned}
\omega \sigma_{1}(\omega)= & 2 \pi t^{2} e^{2} \frac{1}{L} \sum_{|k|<\pi}\left[g_{\pi} \cos ^{2}(k) \delta(\omega-U)\right. \\
& \left.+g_{0} \sin ^{2}(k) \delta(\omega-U-2 \epsilon(k))\right] \\
= & \pi e^{2} t^{2}\left[g_{\pi} \delta(\omega-U)+2 g_{0} /\left(\pi W^{2}\right)\right. \\
& \left.\{[\omega-(U-W)][(U+W)-\omega]\}^{1 / 2}\right] .
\end{aligned}
$$

For the large- $U$ Hubbard model $g_{q}$ cannot be calculated analytically. We can adopt a "no-recoil approximation" 13] to argue that the dominant contributions to the conductivity again come from $q=0$ and $q=\pi$. Figure 11 shows DDMRG results obtained on 64-site lattices for the large- $U$ Hubbard model and the related models with a dimer or Néel-ordered spin ground state. On the scale of this figure there is no visible difference between our numerical results for the two models with spin order and the exact results (舟) with the same broadening $\eta$. This shows that the DDMRG approach is very accurate in these limiting cases.

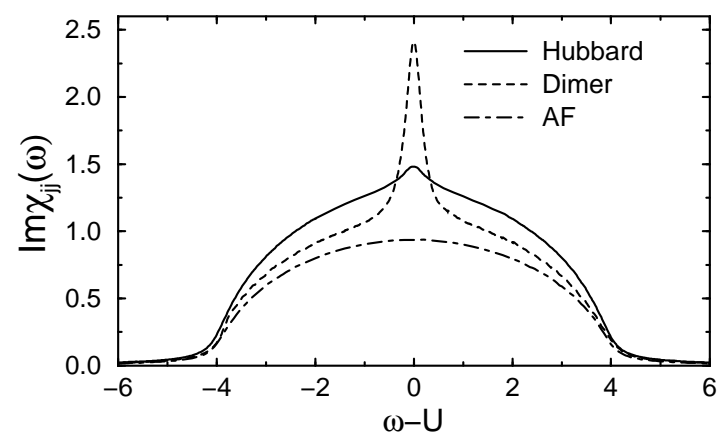

FIG. 1. Current-current correlation function of the large- $U$ Hubbard model $(U / t \rightarrow \infty)$ and of two related models with a dimer and a Néel (AF) spin ground state $(\eta=0.2)$.
For the large- $U$ Hubbard model DDMRG data are also in agreement with eq. (4) confirming the no-recoil approximation in Ref. 13]. In particular, one notes the small bump in $\sigma_{1}(\omega)$ at $\omega=U$ showing that $g_{\pi}>0$ (see Fig. 11). This bump is not visible in small chains but becomes more evident and sharper as we increase the system size. Therefore, there are two salient features in the optical absorption of the large- $U$ Hubbard model, (i), the behavior at threshold is $\sigma_{1}(\omega) \sim \sqrt{\omega-\Delta}$, and (ii), the large density of states for excitations between parallel bands results in a small but visible peak in the middle of the absorption band. The second feature is not present in the optical conductivity calculated using a Néel-ordered spin ground state [13,14 (see also Fig. 11) because the spin form-factor $g_{q}$ of a Néel state vanishes for all $q \neq 0$.

The density-matrix renormalization group (DMRG) is known to be a very accurate numerical method to determine static properties of low-dimensional lattice systems [15,16]. Recently, Kühner and White [11] developed an efficient scheme to calculate dynamical correlation functions in Heisenberg spin chains using DMRG and the correction vector method [12]. We have extended this dynamical DMRG method to correlated electron systems, such as the Hubbard model (11). Our method differs from that of Ref. [11] in two points: (i) we calculate correction vectors with Ramasesha's algorithm [17] instead of a conjugate gradient method, and (ii) we compute dynamical correlation functions directly from the correction vectors.

Our DDMRG method allows us to calculate dynamical correlation functions, such as the r.h.s. of eq. (3a), very accurately for fairly large systems $(L \leq 128)$ and a finite broadening factor $\eta$. Thus, our numerical results always correspond to the actual function $\sigma_{1}(\omega)$ convoluted with a Lorentzian $L(\omega)=\eta /\left[\pi\left(\omega^{2}+\eta^{2}\right)\right]$. We compared the predictions of our method with exact results for a Peierls insulator [13] and the two models with a spin-ordered ground state (4) and found that errors were smaller than $1 \%$ over the entire spectrum. In the limit of small $U / t$ our numerical results agree very well with exact fieldtheory results, see below. We have also systematically checked various sum rules relating moments of the function $\sigma_{1}(\omega)$ to ground-state expectation values that are known exactly or can be evaluated with great accuracy using a ground-state DMRG method [15]. For instance,

$$
\int_{0}^{\infty} \frac{d \omega}{\pi} \sigma_{1}(\omega)=\frac{t}{2 L}\langle 0| \sum_{l ; \sigma}\left(\hat{c}_{l, \sigma}^{+} \hat{c}_{l+1, \sigma}+\text { h.c. }\right)|0\rangle .
$$

Lastly, the optical gaps deduced from DDMRG data always agree with the exact result (2) after taking finitesize corrections into account. 


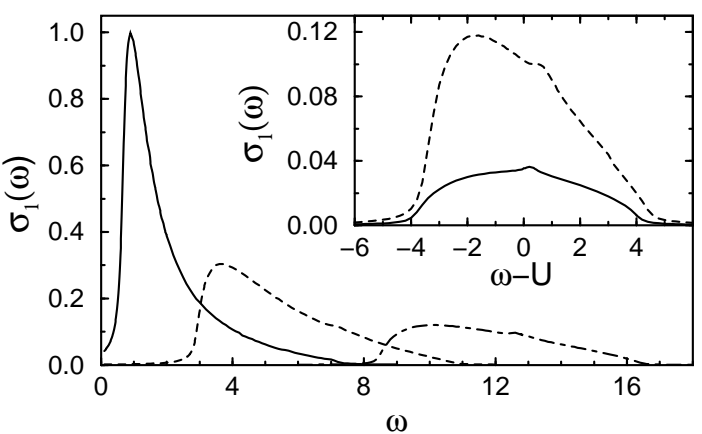

FIG. 2. Optical conductivity for $U / t=3,6,12$ (from left to right), calculated with DDMRG on a 128-site lattice $(\eta=0.1)$. Inset: $\sigma_{1}(\omega)$ for $U / t=12$ (dashed) and 40 (solid) calculated on a 64 -site chain $(\eta=0.2)$.

In Fig. 2 we show the optical conductivity of the Hubbard model calculated with DDMRG for several values of $U / t$. For $U=40 t, \sigma_{1}(\omega)$ resembles the large- $U$ result, compare Fig. 11. As the interaction strength decreases, the width of the absorption spectrum appears to diminish slightly from $8 t$ for the large- $U$ Hubbard model to less than $7 t$ for $U=3 t$. The small peak seen in the large- $U$ Hubbard model is barely visible for $U / t<12$, but this feature actually subsists at least down to $U=4 t$. As seen in Fig. 2, the shape of the optical conductivity changes progressively: for strong coupling $(U=12 t), \sigma_{1}(\omega)$ displays a broad distribution with a maximum clearly above the optical absorption threshold $\omega=\Delta$; for weaker coupling $(U=3 t), \sigma_{1}(\omega)$ develops a very sharp peak close to the threshold and a long tail for higher frequencies. For $U / t \rightarrow 0$ this peak eventually turns into a delta function as all the spectral weight is concentrated in the Drude peak for $U=0$.

For $U / t \geq 3$ the resolution of our calculations is good enough to see that the maximum of $\sigma_{1}(\omega)$ occurs at a frequency significantly above the optical conductivity threshold. Our numerical data are compatible with a square-root increase of the optical conductivity above the Lieb-Wu gap for all values of $U / t \geq 3$, in agreement with the analytical result for $U \gg t$. Obviously, the exact behavior of $\sigma_{1}(\omega)$ at the threshold cannot be determined from the DDMRG results alone because the resolution is limited by the finite broadening $\eta$ and finitesize effects. For the same reasons, it is extremely difficult to study the optical conductivity of the Hubbard chain with DDMRG when the optical gap becomes exponentially small $(U / t<3)$.

For small interaction strengths, $U \ll t$, the half-filled Hubbard model can be mapped [18,19] onto its lowenergy effective field theory, the $\mathrm{SU}(2)$ Thirring model

$$
\mathcal{L}=i \bar{\psi} \not \partial \psi-(U / W) \sum_{a=1}^{3} J_{\mu}^{a} J^{\mu a} .
$$

Here, $\psi$ is a doublet of Dirac spinors and $J_{\mu}^{a}=\frac{1}{2} \bar{\psi} \gamma^{\mu} \sigma^{a} \psi$ are $\mathrm{SU}(2)$ currents, where $\gamma^{\mu}$ are $1+1$ dimensional Dirac matrices and $\sigma^{a}$ are Pauli matrices. The theory (6) decouples into a massless (spin) and massive (charge) sector. The charge sector exhibits a $\mathrm{SU}(2)$ symmetry as is required by the $\mathrm{SO}(4)$ symmetry of the half-filled Hubbard model [20]. This symmetry is in general broken in the Luther-Emery (a.k.a. $U(1)$ Thirring) model [21], and the optical absorption of the half-filled Hubbard model cannot be calculated using the exact solution at the Luther-Emery point [22].

In the field-theory limit, the current operator is found to be proportional to a $\mathrm{SU}(2)$ current $\hat{\jmath} \propto \int d x J_{1}^{3}(x)$. This operator couples only to the gapped charge sector of the theory, so that only (multi) holon-antiholon scattering states contribute to (3b). It can be shown that lattice and band-curvature effects generate a coupling to the spin sector, but this is not important in the field-theory limit. We use the spectral representation of the excited states in terms of scattering states of holons $(h)$, antiholons $(\bar{h})$ and spinons which form a basis of the Hilbert space [23]. Using charge-conjugation symmetry one finds that only states with equal number of holons $N_{h}$ and antiholons $N_{\bar{h}}$ couple to $J_{1}^{3}$.

In the field-theory limit the holon/antiholon dispersion $E(P)=\sqrt{P^{2}+(\Delta / 2)^{2}}$ is parameterized in terms of a rapidity $\theta$ as $E(\theta)=(\Delta / 2) \cosh \theta, P(\theta)=(\Delta / 2) \sinh \theta$. A scattering state of $N$ (anti)holons with rapidities $\left\{\theta_{j}\right\}$ and $\mathrm{SU}(2)$ indices $\left\{\varepsilon_{j}\right\} \quad\left(\varepsilon_{j}=h, \bar{h}\right)$ is denoted by $\left|\theta_{1}, \theta_{2}, \ldots, \theta_{N}\right\rangle_{\varepsilon_{1}, \varepsilon_{2}, \ldots \varepsilon_{N}}$. Its energy and momentum are $E_{N}=\sum_{j=1}^{N} E\left(\theta_{j}\right), P_{N}=\sum_{j=1}^{N} P\left(\theta_{j}\right)$. In this basis, the two-point function of $J_{1}^{3}(x)$ in the spectral representation reads

$$
\begin{aligned}
& \left\langle J_{1}^{3}(t, x) J_{1}^{3}(0,0)\right\rangle=\sum_{n=1}^{\infty} \frac{1}{n !} \sum_{\left\{\varepsilon_{j}\right\}} \int_{-\infty}^{\infty} \prod_{j=1}^{n} \frac{d \theta_{j}}{2 \pi} \\
& \times \exp \left(-i t E_{N}+i x P_{N}\right)\left|\left\langle 0\left|J_{1}^{3}(0)\right| \theta_{1}, \ldots, \theta_{n}\right\rangle_{\varepsilon_{1} \ldots \varepsilon_{n}}\right|^{2} .
\end{aligned}
$$

The matrix elements $\left\langle 0\left|J_{1}^{3}(0)\right| \theta_{1}, \ldots, \theta_{n}\right\rangle_{\varepsilon_{1} \ldots \varepsilon_{n}}$ have been determined in [24], so that we can evaluate (7). An intermediate state with $N_{h}$ holons contributes to $\sigma_{1}(\omega)$ only if $\omega>N_{h} \Delta$. Taking into account only intermediate states with $N_{h}=N_{\bar{h}}=1$ we obtain $(\nu=\omega / \Delta)$

$$
\begin{aligned}
\sigma_{2 \mathrm{p}}(\omega)= & e^{2} C_{2}(\Delta) \frac{2}{\pi} \frac{\sqrt{\nu^{2}-1}}{\nu^{2}} \Theta(\nu-1) \\
& \exp \left(-\int_{0}^{\infty} \frac{d x}{x} \frac{1-\cos (x \theta / \pi) \cosh x}{\exp (x / 2) \cosh (x / 2) \sinh x}\right),
\end{aligned}
$$

where $\theta=2 \operatorname{arccosh}(\nu)$. Formula (8) is exact in the interval $\Delta \leq \omega \leq 2 \Delta$. For $\omega>2 \Delta$ there are corrections to (8), which are due to multi holon/antiholon states and have a more complicated structure, but can be shown to be important only at energies $\omega \gg \Delta$ [25]. At present, the normalization $C_{2}(\Delta)$ cannot be calculated analytically. We note that $S_{2 \mathrm{p}}(\omega) \equiv \sigma_{2 \mathrm{p}}(\omega) / C_{2}(\Delta)$ is a universal function of $\nu=\omega / \Delta . S_{2 \mathrm{p}}(\Delta \leq \omega \leq 2 \Delta)$ is shown in the inset of Fig. 3 . 


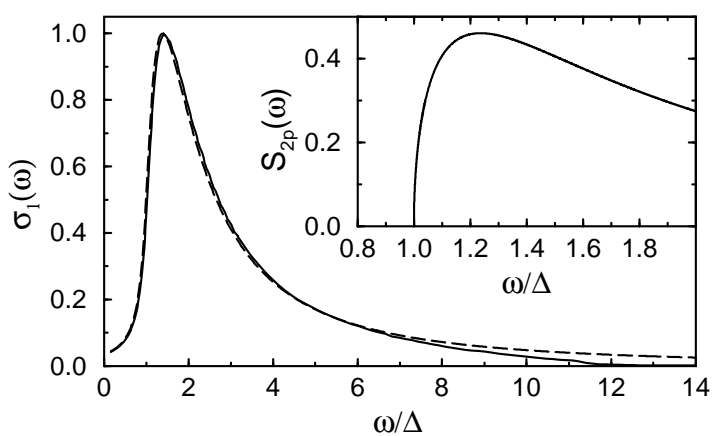

FIG. 3. Optical conductivity for $U / t=3$ from field theory (dashed) and DDMRG (solid). $\sigma_{1}(\omega)$ does not vanish below the gap $\Delta=0.631 t$ because a broadening of $\eta=0.1$ is used. Inset: universal function $S_{2 \mathrm{p}}(\omega)=\sigma_{2 \mathrm{p}}(\omega) / C_{2}(\Delta)$.

$\sigma_{2 \mathrm{p}}(\omega)$ goes through a maximum for $\omega \approx 1.24 \Delta$. For $\omega \rightarrow \Delta$ we find the expected square-root behavior, $\sigma_{1}(\omega \rightarrow \Delta)=\sigma_{2 \mathrm{p}}(\omega \rightarrow \Delta) \sim \sqrt{\omega-\Delta}$. For the Peierls insulator 13, a square-root divergence occurs, reflecting the divergence in the density of states for excitations near the gap. For the Mott-Hubbard insulator, however, this divergence is suppressed by the momentum dependence of the oscillator strengths. For large $U / t$, eq. (4) shows explicitly that $T_{0, n}^{2}(|k| \rightarrow \pi, \omega \gtrsim \Delta) \sim \sin ^{2}(k)$ vanishes quadratically in the vicinity of the gap.

To estimate the normalization $C_{2}(\Delta)$, we use the exact sum rule (5). In the weak-coupling limit $U \ll t$, most of the optical weight of the Hubbard model (11) must be concentrated at low energy $\omega \sim \Delta$ as $\sigma_{1}(\omega)$ reduces to a single Drude peak for $U=0$. Therefore, in the fieldtheory limit, the l.h.s. of (5) can be determined from (8) as the omitted terms have a negligible contribution at low energy. For the r.h.s. we use the exact result at $U=0$. We obtain $C_{2}(\Delta)=1.867 t / \Delta$.

In Fig. 3 we compare the field-theory prediction (8), using the exact value $\Delta \approx 0.631 t$ of the optical gap (2) rather than its field theory value, and the DDMRG result for $U=3 t$. We see that both results agree well up to $\omega \approx 6 \Delta$. This is surprising as the field theory is expected to work only as long as $\Delta \ll t$. It appears that even for $U / t=3$, the contribution of states with more than one holon-antiholon pair to $\sigma_{1}(\omega)$ is almost negligible in this range of frequencies. If we do the same comparison for larger $U / t$, we see that the range of $\omega / \Delta$ for which $\sigma_{2 \mathrm{p}}(\omega)$ matches the DDMRG data becomes smaller, but both methods coincide around the optical absorption threshold even for fairly strong couplings $(U=6 t)$. We have no explanation for this universal behavior of the optical conductivity above $\Delta$.

In conclusion, we have determined the optical conductivity $\sigma_{1}(\omega)$ of the one-dimensional Mott-Hubbard insulator over the entire absorption spectrum for all values of the interaction strength. As also reported in [26], we have found a square-root increase of $\sigma_{1}(\omega)$ at the absorption edge in contrast to the square-root divergence for the one- dimensional Peierls insulator. In principle, this difference could be used to distinguish experimentally between the two types of insulators. However, other interactions, e.g., the long-range parts of the Coulomb interaction, lattice structure, interchain couplings, and disorder, can significantly change the absorption spectrum. We think that both dynamical-DMRG and field-theory approaches will permit the reliable calculation of dynamical properties for more general Hamiltonians which include these additional interactions.

We gratefully acknowledge helpful discussions with D. Baeriswyl, T. Kühner, A.M. Tsvelik, and S.R. White.

[1] Organic Conductors, ed. by J.-P. Farges (Marcel Dekker, New York, 1994).

[2] D. Baeriswyl, D.K. Campbell, and S. Mazumdar in Conjugated Conducting Polymers, ed. by H. Kiess (Springer, Berlin, 1992).

[3] N.F. Mott, Metal-Insulator Transitions, 2nd ed. (Taylor and Francis, London, 1990); F. Gebhard, The Mott Metal-Insulator Transition (Springer, Berlin, 1997).

[4] J. Hubbard, Proc. R. Soc. London A 276, 238 (1963).

[5] E.H. Lieb and F.Y. Wu, Phys. Rev. Lett. 20, 1445 (1968).

[6] A.A. Ovchinnikov, Sov. Phys. JETP 30, 1160 (1970).

[7] F.H.L. Essler and H. Frahm, Phys. Rev. B 60, 8540 (1999).

[8] R.M. Fye et al., Phys. Rev. B 44, 6909 (1991).

[9] N.M.R. Peres et al., Z. Phys. B 103, 217 (1997); N. Tomita and K. Nasu, Phys. Rev. B 56, 3779 (1997).

[10] T. Giamarchi, Phys. Rev. B 44, 2905 (1991); ibid. 46, 342 (1992); Physica B 230-232, 975 (1997).

[11] T.D. Kühner and S.R. White, Phys. Rev. B 60, 335 (1999).

[12] S. Ramasesha et al., Synth. Met. 85, 1019 (1997).

[13] F. Gebhard et al., Phil. Mag. B 75, 1, 13, 47 (1997).

[14] F.B. Gallagher and S. Mazumdar, Phys. Rev. B 56, 15025 (1997).

[15] S.R. White, Phys. Rev. Lett. 69, 2863 (1992); Phys. Rev. B 48, 10345 (1993).

[16] I. Peschel et al., Density-Matrix Renormalization (Springer, Berlin, 1999).

[17] S. Ramasesha, J. Comput. Chem. 11, 545 (1990).

[18] I. Affleck, talk given at the Nato ASI on Physics, Geometry and Topology, Banff, August 1989.

[19] E. Melzer, Nucl. Phys. B443, 553 (1995).

[20] O.J. Heilmann and E.H. Lieb, Ann. N.Y. Acad. Sci. 172, 583 (1971); C.N. Yang, Phys. Rev. Lett. 63, 2144 (1989).

[21] A. Luther and V. Emery, Phys. Rev. Lett. 33, 589 (1974).

[22] A.M. Finkel'shtein, Sov. Phys. JETP Lett. 25, 73 (1977).

[23] F.H.L. Essler and V.E. Korepin, Phys. Rev. Lett. 72, 908 (1994); Nucl. Phys. B426, 505 (1994).

[24] M. Karowski and P. Weisz, Nucl. Phys. B139, 455 (1978); F.A. Smirnov Form Factors in Completely Integrable Models of Quantum Field Theory (World Scientific, Singapore, 1992).

[25] D. Controzzi, F.H.L. Essler, and A.M. Tsvelik, preprint, cond-mat/0005349 (unpublished).

[26] J.M.P. Carmelo, N.M.R. Peres, and P.D. Sacramento, Phys. Rev. Lett. 84, 4673 (2000). 